\title{
Mitochondrial Dysfunction in Type 2 Diabetes-An Update
}

\author{
a report by \\ Muhammad A Abdul-Ghani, MD, PhD' ${ }^{1}$ and Ralph A DeFronzo, MD²
}

\begin{abstract}
1. Assistant Professor of Medicine; 2. Professor of Medicine, and Chief, Division of Diabetes, University of Texas Health Science Center at San Antonio
\end{abstract}
DOI:10.17925/EE.2006.05.00.28

Insulin resistance in skeletal muscle and the liver is a central feature of type 2 diabetes. 'Insulin resistance is also believed to be the underlying mechanism responsible for the metabolic syndrome. ${ }^{1-3}$ Insulin-stimulated glucose disposal in skeletal muscle is reduced in insulin-resistant individuals due to impaired insulin signaling ${ }^{3-5}$ and multiple intracellular defects in glucose metabolism (reviewed in reference 5). Similar defects in insulin signaling have been reported in the liver and adipocytes $^{6}$ and lead to impaired suppression of hepatic glucose production and lipolysis, respectively. ${ }^{7.8}$

Compelling evidence suggests an important role for intracellular deposition of fat in non-adipose tissues, e.g. liver, skeletal, and cardiac muscle, and $\beta$ cell $^{8-14}$ in the pathogenesis of insulin resistance. Both increased exogenous fat intake (obesity) and excess endogenous fat input (accelerated lipolysis, as occurs in obesity and type 2 diabetes) ${ }^{5}$ lead to increased lipid supply to insulin target tissues and excessive lipid accumulation. Alternately, it can be argued that a decrease in oxidative capacity in insulin-responsive tissues is responsible for the increase in intracellular fat content in non-adipose tissues. The intracellular lipid stores are in a state of constant turnover and the accumulation of toxic lipid metabolites, e.g. fatty acyl Co-A (FACOA), ${ }^{11,15,16}$ diacylglycerol $(D A G),{ }^{17}$ and ceramide $^{18}$ produces insulin resistance through the activation of serine kinases, which interfere with the insulin signaling cascade $^{17-23}$ and inhibit multiple intracellular steps involved in glucose

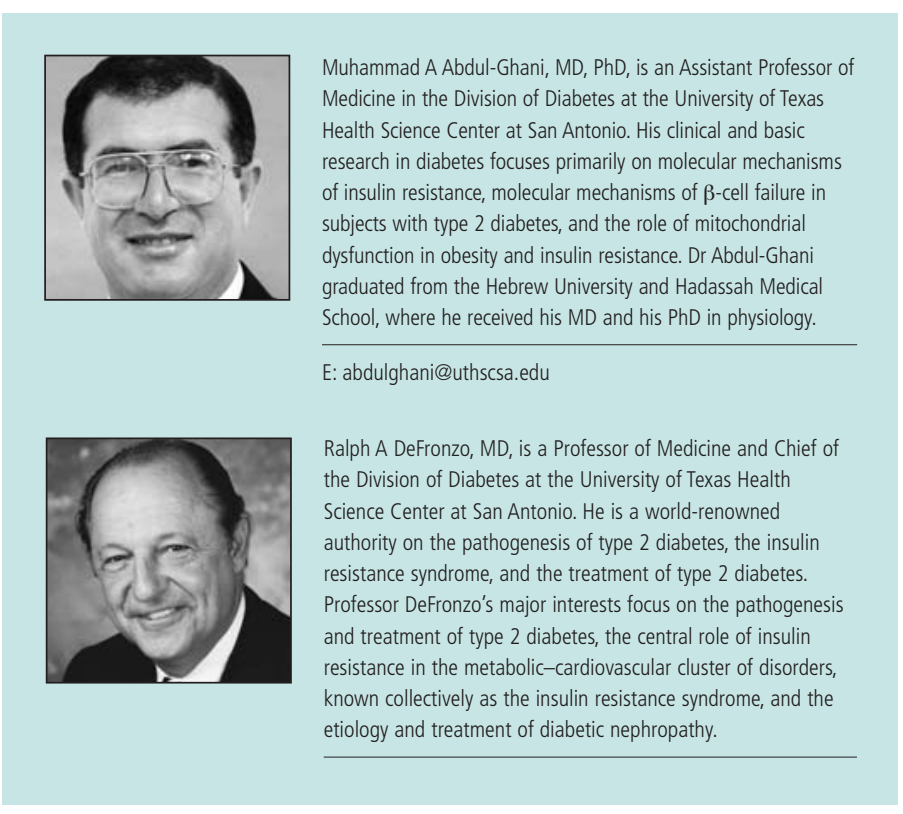

metabolism, including glucose transport and glucose phosphorylation, ${ }^{24,27}$ glycogen synthesis (glycogen synthase), ${ }^{28}$ and glucose oxidation (pyruvate dehydrogenase and Krebs cycle activity). ${ }^{28,29}$ In this article, we will summarize the evidence implicating a possible role for impaired mitochondrial function in the pathogenesis of insulin resistance.

\section{Free Fatty Acid Metabolism and Insulin Resistance}

Due to its accessibility, most studies have examined the relationship between fatty acid metabolism, mitochondrial function, and insulin resistance in skeletal muscle. In subjects with type 2 diabetes and in obese insulin-resistant individuals without diabetes, muscle-fat oxidation is reduced, suggesting an abnormality in mitochondrial oxidative capacity in insulin-resistant individuals. ${ }^{29-33}$ The ability of insulin to suppress lipolysis in insulin-resistant individuals is also impaired ${ }^{7}$ and leads to an increase in the plasma free fatty acid (FFA) concentration and enhanced FFA influx into the skeletal muscle. In the presence of impaired mitochondrial fat oxidation, an increased FFA influx could explain the elevated intramyocellar fat content and increase in intramyocellar longchain FACOA, diacylglycerol (DAG), and ceramide concentrations observed in type 2 diabetes and obese individuals without diabetes. ${ }^{15-18}$ Increased levels of these toxic lipid metabolites, through serine phosphorylation of major molecules in the insulin signaling pathway, would impair insulin action and lead to insulin resistance. Thus, an inherited or acquired mitochondrial defect, in combination with increased fat supply to non-adipose tissues, could explain the link between increased plasma FFA levels, the accumulation of intramyocellar lipids, and insulin resistance.

Mitochondrial Function in Insulin Resistance Disorders Insulin resistance is the hallmark of type 2 diabetes and obesity, but it is also a characteristic feature of the normal aging process and other common clinical conditions, including the metabolic syndrome, polycystic ovarian syndrome, and non-alchoholic steatohepatitis. In vivo measurement of oxidative phosphorylation with ${ }^{31}$ P-NMR has demonstrated impaired adenosine triphosphate (ATP) synthesis in a variety of insulin-resistant states. Subjects with type 2 diabetes, ${ }^{34-36}$ lean to normal glucose-tolerant elderly individuals, ${ }^{37}$ lean normal-glucose-tolerant (NGT) insulin-resistant offspring of patients with type 2 diabetes, ${ }^{38}$ and healthy young subjects in whom insulin resistance was induced with lipid infusion ${ }^{39}$ all manifest a $30-40 \%$ decrease in oxidative phosphorylation measured with magnetic resonance spectroscopy (MRS). Furthermore, the defect in oxidative phosphorylation in lean, NGT, insulin-resistant offspring of patients with type 2 diabetes is associated with a decreased metabolic flux through the tricarboxylic acid (TCA) cycle under basal conditions. ${ }^{40}$ Unlike 
lean insulin-sensitive individuals, both patients with type 2 diabetes and NGT insulin-resistant offspring of two parents with diabetes fail to increase mitochondrial oxidative phosphorylation flux normally following insulin stimulation, despite a significant increase in glucose disposal in skeletal muscle. ${ }^{34,35}$ Several studies have reported that patients with type 2 diabetes have impaired recovery of intracellular phosphocreatinine concentration following exercise, ${ }^{36,41,42}$ suggesting that a mitochondrial defect in oxidative phosphorylation contributes to the impairment in exercise capacity in these insulin-resistant individuals. One study ${ }^{43}$ reported that patients with type 2 diabetes had a similar phosphocreatinine recovery rate following exercise to obese NGT individuals. However, insulin sensitivity was not measured in that study, and as obese NGT subjects would be expected to have increased insulin resistance in skeletal muscle, the decreased rate of phosphocreatinine recovery may reflect their increased level of insulin resistance. Collectively, the results reviewed above indicate that, regardless of its etiology, insulin resistance in skeletal muscle is associated with decreased mitochondrial oxidative phosphorylation.

\section{Characteristics of the Mitochondrial Defect in Insulin-resistance States}

The studies reviewed above indicate that insulin resistance in skeletal muscle is associated with decreased mitochondrial oxidative phosphorylation. However, they do not provide information about the mechanism of the defect. Furthermore, because of the cross-sectional design of the studies, it is impossible to determine the cause-effect relationship between insulin resistance and mitochondrial dysfunction. A decrease in the metabolic flux, e.g. due to reduced substrate availability, without a change in the mitochondrial oxidative capacity could lead to the decrease in oxidative phosphorylation flux rate documented in vivo with MRS. Conversely, a primary or acquired defect in mitochondrial oxidative capacity could also explain the in vivo MRS observations. A number of abnormalities have been implicated in the reduced muscle mitochondrial oxidative capacity, i.e. a reduction in the number of mitochondria in skeletal muscle with normal function of individual mitochondria, an intrinsic defect in mitochondrial function without any change in mitochondrial number, or a combination of the two. All three of these scenarios would result in a reduced rate of ATP synthesis measured with MRS. Another important unanswered question is whether the mitochondrial defect is inherited or acquired. An acquired defect could be potentially reversed or prevented, while an inherited defect is unlikely to be reversible.

Electron microscopic studies have revealed a significant reduction ( $40 \%)$ in mitochondrial density in skeletal muscle in a variety of insulin resistance groups, including the lean offspring of parents with type 2 diabetes, obese individuals without diabetes, and patients with type 2 diabetes. ${ }^{19,44}$ However, studies that have assessed mitochondrial copy number in skeletal muscle in insulin-resistant individuals have reported inconsistent results. ${ }^{45-47}$ The decrease in mitochondrial density in skeletal muscle in insulin-resistant individuals is consistent with decreased expression of PGC-1, the master regulator for mitochondrial biogenesis, and multiple other genes involved in mitochondrial oxidative phosphorylation..$^{48-50}$

Morphological and functional studies have also provided support for an intrinsic mitochondrial defect in insulin-resistant individuals. Decreased activity of a variety of mitochondrial enzymes has been reported in patients with type 2 diabetes compared with age-matched healthy individuals. ${ }^{51} \mathrm{~A}$ variety of mitochondrial morphological abnormalities have been described in insulin-resistant individuals utilizing electron microscopy (EM) techniques, ${ }^{52}$ and some of these changes could be reversed, at least in part, with weight loss and increased physical activity..$^{53}$

Ex vivo measurements of mitochondrial function have also demonstrated functional as well as quantitative defects in skeletal muscle mitochondria. Decreased electron transport chain activity $(E T C)^{44}$ and reduced mitochondrial ATP synthesis in isolated mitochondria ${ }^{54}$ have been reported in obese insulin-resistant individuals. Utilizing permeabilized muscle fibers, two studies reported a decrease in oxygen consumption in mitochondria obtained from patients with type 2 diabetes compared with age- and body mass index (BMI)-matched healthy subjects ${ }^{43,47}$ However, in the one study in which it was measured, the messenger DNA (mDNA) copy number was found to be decreased and, after normalizing oxygen consumption to mDNA copy number, subjects with type 2 diabetes had a similar oxygen consumption rate compared to healthy controls,${ }^{47}$ suggesting a decrease in mitochondrial density with normal mitochondrial function.

Considerable evidence also supports an acquired defect in mitochondrial function in insulin-resistant states such as type 2 diabetes and obesity. Mitochondrial ATP synthesis rate, measured in vivo with ${ }^{31} \mathrm{P}-\mathrm{NMR}$, strongly and inversely correlates with the fasting plasma FFA concentration, ${ }^{34}$ suggesting a possible role for elevated plasma FFA/muscle fat content in the mitochondrial dysfunction in insulin-resistant individuals. Consistent with this scenario, short-term (48-72 hours) physiological elevation of the plasma FFA concentration in lean healthy individuals has also been shown to decrease the expression of PGC-1 and numerous other mitochondrial genes involved in oxidative phosphorylations. ${ }^{55}$

\author{
Decreased activity of a variety of \\ mitochondrial enzymes has been reported \\ in patients with type 2 diabetes compared \\ with age-matched healthy individuals.
}

Most recently, we have demonstrated that an increase in FACOA concentration inhibits mitochondrial ATP synthesis in vitro in mitochondria isolated from skeletal muscle of NGT healthy lean subjects. ${ }^{56}$ This observation is consistent with the decline in oxidative phosphorylation $^{39}$ and the decreased expression of genes encoding for mitochondrial protein ${ }^{55}$ observed following an elevation in the plasma FFA concentration in lean healthy individuals, and provides strong evidence that an elevation in plasma FFA concentration can cause an acquired mitochondrial defect in oxidative phosphorylation.

In summary, an inherited defect in mitochondrial function, with or without a decrease in mitochondrial density, would be expected to result in impaired lipid oxidation, an increase in intramyocellular lipid content, and the development/exacerbation of insulin resistance. Conversely, an increase in fatty acid flux into the mitochondria, e.g. secondary to obesity 
and tissue fat overload or adipocyte resistance to the antilipolytic effect of insulin, could lead to an acquired defect in mitochondrial function. There is experimental evidence to support both of these scenarios, which are not mutually exclusive.

\section{Discordance Between Insulin Resistance and Mitochondrial Function}

Due to the cross-sectional design of the above studies, it is impossible to establish causality between mitochondrial dysfunction and insulin resistance in skeletal muscle. Studies in experimental animals have altered mitochondrial function in skeletal muscle and assessed the impact of this intervention on the subsequent development of muscle insulin resistance. Unfortunately, these results have yielded conflicting results. Overexpression of the PGC-1 alpha gene in skeletal muscle in mice in vivo enhanced mitochondrial activity, augmented the expression of multiple proteins involved in fat oxidation and glucose transport, and increased by $\sim 35 \%$ insulin-stimulated glucose uptake in skeletal muscle. ${ }^{57}$ Similarly, activation of sirtuin 1 (SIRT1) with resveratrol in mice increased mitochondrial function and protected the animal from diet-induced obesity and insulin resistance. ${ }^{58}$ Although these studies indicate that, in animal models, increasing mitochondrial oxidative capacity has a favorable effect on insulin sensitivity, a decrease in mitochondrial oxidative capacity in skeletal muscle has also been shown to improve insulin sensitivity. Popisilik et al. reported that a reduction in mitochondrial oxidative capacity, brought about by knocking down apoptosis-initiating factor (AIF), resulted in enhanced insulin sensitivity and protection from fat-induced insulin resistance. ${ }^{59}$ The results of these genetic manipulations should be interpreted with caution, as they represent extreme situations where a complete lack of or marked overexpression of a protein is present. Therefore, they do not reproduce the physiological situation and are likely to trigger other compensatory mechanisms. For example, the impact of PGC-1 alpha overexpression on insulin sensitivity in skeletal muscle is time- and magnitude-dependent. 57,60 Moreover, these genetic manipulations may have additional direct effects on insulin action that are independent of changes in mitochondrial function.
In patients with type 2 diabetes, interventions aimed at decreasing insulin resistance with weight loss and increased physical activity or with thiazolidinedions have also yielded inconsistent results. While weight loss and increased physical activity were associated with increased oxidative capacity and reversal of the mitochondrial morphological changes associated with insulin resistance, ${ }^{61,62}$ the decrease in insulin resistance caused by caloric restriction ${ }^{61}$ or with rosiglitazone ${ }^{63}$ was not associated with any improvement in mitochondrial function. These conflicting results suggest that factors other than mitochondrial dysfunction contribute to the development of insulin resistance in subjects with type 2 diabetes.

\section{Summary and Conclusions}

Although there is appealing evidence from both in vivo and ex vivo human studies to implicate a mitochondrial defect (reduced mitochondrial number and/or function) in oxidative phosphorylation with the development of skeletal muscle increased insulin resistance, the cause-effect relationships between insulin resistance and mitochondrial dysfunction remain to be defined. Furthermore, the nature of the mitochondrial defect, i.e. quantitative versus functional, remains to be definitively established. Such a mitochondrial defect, in the face of excessive endogenous (increased lipolysis) or exogenous (overfeeding and obesity) FFA supply, would be expected to lead to the accumulation of fat in insulin-sensitive tissues, e.g. skeletal muscle and liver, resulting in the development of insulin resistance. The cause-effect relationship between insulin resistance and mitochondrial dysfunction can be examined in longitudinal intervention studies.

A major challenge is to determine whether, in light of the enormous potential to increase skeletal muscle mitochondrial oxidative capacity that can be observed during exercise, a $\sim 30 \%$ decrease in mitochondrial function measured in vivo during the resting state can lead to an increase in intramyocellar fat content. It also remains to be determined whether strategies that specifically upregulate mitochondrial function in skeletal muscle have a therapeutic potential to decrease insulin resistance and improve glucose tolerance in patients with type 2 diabetes.
1. DeFronzo RA, Banting Lecture. From the Triumvirate to the Ominous Octet: A new paradigm for the treatment of type 2 diabetes, Diabetes, 2008; in press.

2 Reaven GM, Banting lecture. Role of insulin resistance in human disease, Diabetes, 1988;37:1595-1607.

3. Bajaj M, Defronzo RA, Metabolic and molecular basis of insulin resistance, J Nucl Cardiol, 2003;10:311-23.

4. Cusi K, Maezono K, Osman A, et al., Insulin resistance differentially affects the PI 3-kinase-and MAP kinase-mediated signaling in human muscle, J Clin Invest, 2000;105:311-20.

5. Defronzo RA, Pathogenesis of type 2 diabetes mellitus: metabolic and molecular implications for identifying diabetes genes, Diabetes Rev, 1007;5:117-69.

6. Suzuki R, Tobe K, Aoyama M, et al., Both insulin signaling defects in the liver and obesity contribute to insulin resistance and cause diabetes in Irs2(--. mice, J Biol Chem, 2004;279:25039-49.

7. Groop LC, et al., Glucose and free fatty acid metabolism in noninsulin-dependent diabetes mellitus. Evidence for multiple sites of insulin resistance, I Clin Invest, 1989; 84:205-13.

8. Groop LC, Saloranta C, Shank M, et al., The role of free fatty acid metabolism in the pathogenesis of insulin resistance in obesity and noninsulin-dependent diabetes mellitus, I Clin Endocrinol Metab, 1991;72:96-107.

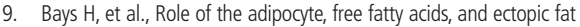
in pathogenesis of type 2 diabetes mellitus: peroxisomal proliferator- activated receptor agonists provide a rational therapeutic approach, J Clin Endocrinol Metab, 2004;89:463-78.

10. Belfort $R$, Harrison $S A$, Brown $K$, et al., A placebo controlled trial of pioglitazone in patients with non-alcoholic steatohepatitis, NEJM, 2006;355:2297-2307.

11. Bajaj M, Suraamornkul S, Romanelli A, et al., Effect of sustained reduction in plasma free fatty acid concentration on intramuscular long chain-fatty acyl-CoAs and insulin action in patients with type 2 diabetes, Diabetes, 2005;54:3148-53.

12. Mayerson $A B$, Hundal RS, Dufour $S$, et al., The effects of rosiglitazone on insulin sensitivity, lipolysis, and hepatic and skeletal muscle triglyceride content in patients with type 2 diabetes, Diabetes, 2002:51:797-802

13. Sharma S, Adrogue JV, Golfman L, et al., Intramyocardial lipid accumulation in the failing human heart resembles the liptoxic rat heart, FASEB, 2004;18:692-700

14. Kim JK, Fillmore JJ, Chen Y, et al., Tissue-specific overexpression of lipoprotein lipase causes tissue-specific insulin resistance, Proc Natl Acad Sci U S A, 2001;98:7522-7.

15. Houmard JA, Tanner CJ, Yu C, et al., Effect of weight loss on insulin sensitivity and intramuscular long-chain fatty acyl-CoAs in morbidly obese subjects, Diabetes, 2002;51:2959-63.

16. Ellis BA, et al., Long-chain acyl-CoA esters as indicators of lipid metabolism and insulin sensitivity in rat and human muscle, Am $J$ Physiol Endocrinol Metab, 2000;279:E554-60.
17. Itani SI, Ruderman NB, et al., Lipid-induced insulin resistance in human muscle is associated with changes in diacylglycerol, protein kinase C, and IB-, Diabetes, 2002;51: 2005-11.

18. Adams JM II, Pratipanawatr T, Berria R, et al., Ceramide content is increased in skeletal muscle from obese insulin-resistant humans, Diabetes, 2004;53:25-31.

19. Morino K, Petersen KF, Dufour S, et al., Reduced mitochondrial density and increased IRS-1 serine phosphorylation in muscle of insulin-resistant offspring of type 2 diabetic parents, J Clin Invest, 2005;115:3587-93.

20. Griffin ME, Marcucci MJ, Cline GW, et al., Free fatty acid-induced insulin resistance is associated with activation of protein kinase $C$ theta and alterations in the insulin signaling cascade, Diabetes, 1999:48:1270-74.

21. Dresner $A$, Laurent $D$, Marcucci $M$, et al., Effects of free fatty acids on glucose transport and IRS-1-associated phosphatidylinositol 3-kinase activity, J Clin Invest, 1999;103:253-9.

22. Yu C, Chen Y, Cline GW, et al., Mechanism by which fatty acids inhibit insulin activation of insulin receptor substrate-1 (IRS-1)associated phosphatidylinositol 3-kinase activity in muscle, I Biol Chem, 2002;277:50230-36.

23. Miyazaki Y, He H, Mandarino LJ, DeFronzo RA, Rosiglitazone improves downstream insulin-receptor signaling in type 2 diabetic patients, Diabetes, 2003;52:1943-50.

24. Pendergrass M, Bertoldo A, Bonadonna R, et al., Muscle glucose 
transport and phosphorylation in type 2 diabetic, obese non-diabetic, and genetically predisposed individuals, Am J Physiol Endocrino Metab, 2007;292:E92-100.

25. Thompson AL, Cooney GJ, Acyl-CoA inhibition of hexokinase in rat and human skeletal muscle is a potential mechanism of lipid-induced insulin resistance, Diabetes, 2000:49:1761-4.

26. Tippett $P S$, Neet $K E, A n$ allosteric model for the inhibition of glucokinase by long chain acyl coenzyme A, J Biol Chem, 1982; 257:14846-52.

27. Rothman DL, Magnusson I, Cline G, et al., Decreased muscle glucose transport/phosphorylation is an early defect in the pathogenesis of non-insulin-dependent diabetes mellitus, Proc Natl Acad Sci U S A, 1995:92:983-7.

28. Wititsuwannakul D, Kim K, Mechanism of palmityl coenzyme A inhibition of liver glycogen synthase $J$ J Biol Chem, 1977;252: 7812-17

29. Randle PF, Garland PB, Hales CN, Newsholme EA, The glucose fatty acid cycle: its role in insulin sensitivity and the metabolic disturbances of diabetes mellitus, Lancet, 1963;1:785-9.

30. Kelley D, Mandarino L, Fuel selection in human skeletal muscle in insulin resistance: a reexamination, Diabetes, 2000;49:677-83.

31. Kelley $D E$, Simoneau JA, Impaired free fatty acid utilization by skeletal muscle in non-insulin-dependent diabetes mellitus, J Clin Invest, 1994;94:2349-56.

32. Cha BS, Ciaraldi TP, Park KS, et al., Impaired fatty acid metabolism in type 2 diabetic skeletal muscle cells is reversed by PPARgamma agonists, Am J Physiol Endocrinol Metab, 2005;289:E151-E159.

33. Thyfault JP, Kraus RM, Hickner RC, et al., Impaired plasma fatty acid oxidation in extremely obese women, Am J Physiol Endocrinol Metab, 2004;287:E1076-E81.

34. Kim JY, Hickner RC, Cortright RL, et al., Lipid oxidation is reduced in obese human skeletal muscle, Am J Physiol Endocrinol Metab, 2000;279:E1039-E1044.

35. Szendroedi J, Schmid Al, Chmelik M, et al., Muscle mitochondrial ATP synthesis and glucose transport/phosphorylation in type 2 diabetes, PLoS Med, 2007:4:0858-0867.

36. Petersen KF, Dufour $\mathrm{S}$, et al., Decreased insulin-stimulated ATP synthesis and phosphate transport in muscle of insulin-resistant offspring of type 2 diabetic parents, PLoS Med, 3005; 2:e233.

37. Scheuermann-Freestone M, Madsen PL, Manners D, et al., Abnormal cardiac and skeletal muscle energy metabolism in patients with type 2 diabetes, Circulation, 2003;107:3040-46

38. Petersen $\mathrm{KF}$, et al., Mitochondrial dysfunction in the elderly: possible role in insulin resistance, Science, 2003; 300:1140-42.

39. Petersen KF, Dufour S, Befroy D, et al., Impaired mitochondrial activity in the insulin-resistant offspring of patients with type 2 diabetes, $N$ Engl I Med, 2004:350:664-71.

40. Brehm A, Krssak M, Schmid Al, et al., Increased lipid availability impairs insulin-stimulated ATP synthesis in human skeletal muscle, Diabetes, 2006;55:136-40.

41. Befroy DE, Petersen $K F$, Dufour $\mathrm{S}$, et al., Impaired mitochondrial substrate oxidation in muscle of insulin-resistant offspring of type 2 diabetic patients, Diabetes, 2007:56:1376-81.

42. Schrauwen-Hinderling VB, Kooi ME, Hesselink MK, et al., Impaired in vivo mitochondrial function but similar intramyocellular lipid content in patients with type 2 diabetes mellitus and BMI-matched control subjects, Diabetologia, 2007;50:113-20.

43. Phielix E, Schrauwen-Hinderling VB, Mensink M, et al., Lower intrinsic ADP-stimulated mitochondrial respiration underlies in vivo mitochondrial dysfunction in muscle of male type 2 diabetic patients, Diabetes, 2008;57(11):2943-9.

44. De Feyter HM, et al., Early or advanced stage type 2 diabetes is not accompanied by in vivo skeletal muscle mitochondrial dysfunction, Eur J Endocrinol, 2008;158:643-53.

45. Ritov VB, Menshikova EV, He J, et al., Deficiency of subsarcolemmal mitochondria in obesity and type 2 diabetes, Diabetes, 2005;54:8-14.

46. Rolo AP, Palmeira CM, Diabetes and mitochondrial function: role of hyperglycemia and oxidative stress, Toxicol Appl Pharmacol, 2006:212:167-78.

47. Boushel R, Gnaiger E, Schjerling $P$, et al., Patients with type 2 diabetes have normal mitochondrial function in skeletal muscle, Diabetologia, 2007;50:790-96.

48. Asmann YW, Stump CS, Short KR, et al., Skeletal muscle mitochondrial functions, mitochondrial DNA copy numbers, and gen transcript profiles in type 2 diabetic and nondiabetic subjects at equal levels of low or high insulin and euglycemia, Diabetes, 2006;55:3309-19.

49. Patti ME, Butte AJ, Crunkhorn S, et al., Coordinated reduction of genes of oxidative metabolism in humans with insulin resistance and diabetes: Potential role of PGC1 and NRF1, Proc Natl Acad Sci U S A 2003;100:8466-71.

50. Mootha VK, Lindren CM, Eriksson KF, et al., PGC-1 alpha-responsive genes involved in oxidative phosphorylation are coordinately downregulated in human diabetes, Nature Gen, 2003;34:267-73.

51. Mogensen $M$, Sahlin $K$, Fernstrom M, et al., Mitochondrial respiration is decreased in skeletal muscle of patients with type 2 diabetes, Diabetes, 2007:56:159-9.

52. Kelley DE, He J, Menshikova EV, Ritov VB, Dysfunction of mitochondria in human skeletal muscle in type 2 diabetes, Diabetes, 2002; 51:2944-50.

53. Toledo FG, Watkins S, Kelley DE, Changes induced by physical activity and weight loss in the morphology of intermyofibrillar mitochondria in obese men and women, J Clin Endocrinol Metab, 2006;91:3224-7.

54. Abdul-Ghani MA, Jani R, Chavez A, et al., Mitochondrial reactive oxygen species generation in obese non-diabetic and T2DM subjects, Diabetologia, 2009; in press.

55. Richardson DK, Kashyap S, Bajaj M, et al., Lipid infusion induces an inflammatory/fibrotic response and decreases expression of nuclear encoded mitochondrial genes in human skeletal muscle, J Biol Chem, 2005;280:10290-97.

56. Abdul-Ghani MA, Muller F, Liu Y, et al., Deleterious effect of elevated fatty acid metabolites concentration on skeletal muscle mitochondria ATP synthesis, Am J Physiol Endocrinol Metab, 2008;295:E678-85.

57. Benton CR, Nickerson JG, Lally J, et al., Modest PGC-1alpha overexpression in muscle in vivo is sufficient to increase insulin sensitivity and palmitate oxidation in SS, not IMF, mitochondria, J Biol Chem, 2008;283(7):4228-40.

58. Lagouge M, Argmann C, et al., Resveratrol improves mitochondria function and protects against metabolic disease by activating SIRT 1 and PGC-1alpha, Cell, 2006;127: 1109-22.

59. Pospisilik JA, Knauf C, Joza N, et al., Targeted deletion of AIF decreases mitochondrial oxidative phosphorylation and protects from obesity and diabetes, Cell, 2007;131:476-91.

60. Calvo JA, Daniels TG, Wang X, et al., Muscle-specific expression of PPAR $\gamma$ coactivator- $1 \alpha$ improves exercise performance and increases peak oxygen uptake, J Appl Physiol, 2008;104:1304-12.

61. Menshikova EV, Ritov VB, Toledo FG, et al., Effects of weight loss and physical activity on skeletal muscle mitochondrial function in obesity, Am J Physiol Endocrinol Metab, 2005;288:E818-25.

62. Toledo FG, Menshikova EV, Azuma K, et al., Mitochondrial capacity in skeletal muscle is not stimulated by weight loss despite increases in insulin action and decreases in intramyocellular lipid content, Diabetes, 2008:57:987-94.

63. Schrauwen-Hinderling VB, et al., The insulin sensitizing effect of rosiglitazone in type 2 diabetes mellitus patients does not require improved in vivo muscle mitochondrial function, I Clin Endocrinol Metab, 2008:93: 2917-21.

\section{By the Same Authors}

Strong Association Between Insulin Resistance in Liver and Skeletal Muscle in Non-diabetic Subjects

Abdul-Ghani MA, Matsuda M, DeFronzo RA

Diabet Med, 2008;25(11):1289-94.

This study sought to examine the association between insulin resistance in the skeletal muscle and the liver in subjects without diabetes. The study population included a total of 182 Mexican American subjects without type 2 diabetes who underwent an oral glucose tolerance test and euglycaemic-hyperinsulinaemic clamp performed with $[3 \mathrm{H}]$ glucose. Hepatic insulin resistance was strongly correlated $(r=0.68$, $\mathrm{p}<0.0001$ ) with skeletal muscle insulin resistance. Thirty-eight percent of subjects had increased insulin resistance in both liver and skeletal muscle, while $39 \%$ were insulin-sensitive in both skeletal muscle and liver. The study concluded that in subjects without diabetes, insulin resistance in skeletal muscle is an important determinant of fasting and two-hoour plasma glucose concentrations and strongly correlates with hepatic insulin resistance.
Fasting versus Post-load Plasma Glucose

Concentration and the Risk for Future Type 2

Diabetes: Results from the Botnia Study

Abdul-Ghani MA, Lyssenko V, Tuomi T, DeFronzo RA, Groop L

Diabetes Care, 2009:32(2):281-6.

The purpose of this study was to assess the efficacy of post-load plasma glucose concentration in predicting future risk for type 2 diabetes compared with prediction models based on measurement of the fasting plasma glucose concentration. A total of 2,442 subjects from the Botnia Study who were free of type 2 diabetes at baseline received an oral glucose tolerance test (OGTT) at baseline and after seven to eight years of follow-up. The study concluded that plasma glucose concentration at one hour during the OGTT is a strong predictor of future risk for type 2 diabetes and adds to the prediction power of models based on measurements made during the fasting state. A plasma glucose cutpoint of $155 \mathrm{mg} / \mathrm{dl}$ plus the Adult Treatment Panel III criteria for the metabolic syndrome can be used to stratify subjects without diabetes into low-, intermediate-, and high-risk groups. 